\title{
Inhibition of oestrogen-induced nest-building in the canary (Serinus canarius) by stimuli from the nest
}

\author{
Elizabeth Steel and R. A. Hinde \\ M.R.C. Unit on the Development and Integration of Behaviour, \\ Madingley, Cambridge, CB3 $8 A A, U . K$.
}

In the natural breeding season stimuli from the nest which the female canary has constructed are instrumental in bringing nest-building to an end shortly before the first egg is laid, but active nestbuilding can be reduced at any time by substitution of an artificially constructed nest with a small internal diameter and grassy texture for the standard plastic canary nest-pan (Hinde, 1958). Conversely, if the material placed daily in the nest by the female is constantly removed nest-building continues for long periods at a high level (Hinde, 1965). Tactile stimuli from the nest are received by skin receptors in the ventral brood patch; this develops, under the influence of oestrogen and other hormones, during the pre-laying period and involves local defeathering, and an increase in vascularity and in sensitivity to tactile stimulation (Hinde, 1962; Hinde, Bell \& Steel, 1963). The question arises whether, in an intact female, tactile stimulation reduces nest-building by lowering ovarian oestrogen production or by some other means such as an alteration in the responsiveness of central behaviourcontrolling mechanisms to continuing high oestrogen levels.

Nest-building behaviour can be induced in female canaries by exogenous oestrogen (Warren \& Hinde, 1959) and in ovariectomized oestrogen-treated birds the nest-building response to a given amount of exogenous oestrogen is affected by various external stimuli. The roles of daylength (Hinde, Steel \& Follett, 1974) and of male song (Hinde \& Steel, 1976) as factors increasing the response to oestrogen have been studied in some detail. We have also noted (Hinde, Steel \& Follett, 1974, and unpublished observations) that nest-building may decline in the presence of continued high levels of exogenous oestrogen and may even be replaced by a new behaviour pattern-that of incubation. This raises the possibility that, just as the above-mentioned external stimuli affect the appearance and amount of building induced by oestrogen, so further stimuli may affect the cessation of building in the presence of continued oestrogen administration. This paper investigates the role of the nest in this context.

Twenty ovariectomized female canaries (Serinus canarius) were kept in a $14 \mathrm{~h}$ light $/ 24 \mathrm{~h}$ schedule and injected thrice weekly for 5 weeks with $50 \mu \mathrm{g}$ oestradiol benzoate in $50 \mu 10.9 \%(\mathrm{w} / \mathrm{v}) \mathrm{NaCl}$. All birds had experienced oestrogen-induced nest-building since ovariectomy but at least 2 months had elapsed since the last oestrogen injection. The birds were provided with dispensers full of $10-\mathrm{cm}$ lengths of string and felt-lined nest pans in which to build a nest. For the first 26 days of the experiment daily records were made of the weight of the string placed in the nest pan. Ten birds had the nest material removed daily (Group 1), while 10 birds were left with their nest undisturbed (Group 2). These latter nests were weighed daily and the weight of the nest pan was subtracted from the total weight to give that of the amount of string added without disturbance to the nest.

Mann-Whitney $U$ tests were used to compare the groups for various measures of nest-building. All probabilities are one-tailed.

There was no significant difference between the groups in the latency to the first day on which placing occurred. The median was 11 (range 4-28) days for the Group 1 birds and 8.5 (3-36) days for the Group 2 birds.

Daily records of placing were available up to Day 26; by this day 7 birds had placed over $1 \mathrm{~g}$ material. The total placed by Day 26 was significantly different between the two groups. Birds in Group 1 tended to place material on more days than did those in Group 2 and the mean amount placed (per day of placing) was higher (Table 1). The typical pattern of building in Group 2 was to build actively on 1 or 2 days and subsequently add or remove only a few strings to/from the nest. By 
contrast, the birds in Group 1 placed actively on a larger number of days with wide fluctuations from day to day.

Table 1. Various measures of nest-building (medians and interquartile ranges) on Days 2-26 for the 7 most active builders in two groups of oestrogen-treated ovariectomized canaries for which the nest material was either removed daily from the nest pan (Group 1) or left undisturbed (Group 2)

\begin{tabular}{|c|c|c|c|}
\hline & Group 1 & Group 2 & Significance \\
\hline Total amount of material placed $(\mathrm{g})$ & $74(27-166)$ & $30(19-33)$ & $P<0.05$ \\
\hline $\begin{array}{l}\text { No. of days when placing occurred } \\
\text { Mean amount of material placed// }\end{array}$ & $14(11-16)$ & $11(9-13)$ & $0.1>P>0.05$ \\
\hline day of placing $(\mathrm{g})$ & $5 \cdot 3(1.9-11 \cdot 9)$ & $2 \cdot 7(1 \cdot 3-4 \cdot 2)$ & $0.1>P>0.05$ \\
\hline
\end{tabular}

These differences in the general patterns of building activity are reflected in a number of specific measures. As shown in Text-fig. 1, the highest daily level of placing was followed by a day of low placing activity whether the material was removed or retained. On the 2 nd day after the peak, placing activity recovered only slightly in Group 2, but birds in Group 1 resumed placing at levels not far below those of the peak day. The difference between the groups on Day +2 was significant $(P<0.01)$.

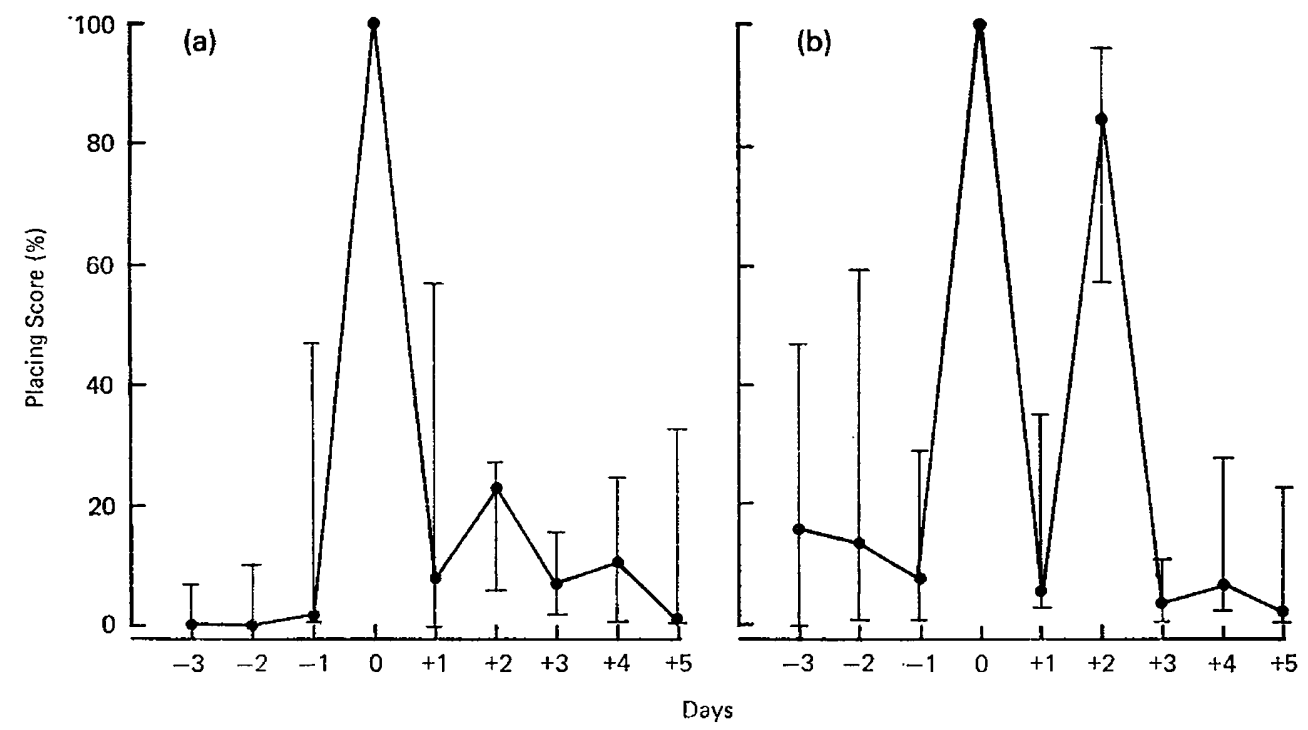

Text-fig. 1. Peak placing scores (expressed as $100 \%$ ) for each bird are superimposed on Day 0 . Placing scores of Days -3 to +5 are expressed as percentages of the Day 0 placing score. Medians and interquartile ranges are plotted. (a) Group 2 (nest retained), $N=7$; (b) Group 1 (nest removed) $N=7$.

The highest placing scores came significantly $(P<0.001)$ earlier in the experiment in Group 2 (median 8 days) than in Group 1 (median 17 days). The accumulated nest depressed subsequent building activity while birds whose nest was removed daily continued (after the initial inhibition) to place on many subsequent days. During the last week of the experiment (Days 27-33) the birds in Group 1 continued to build actively and added a median of $62 \mathrm{~g}$ to the nest (range 1-115 g), while those in Group 2 tended to remove rather than to add material (median $0 \mathrm{~g}$; range $-0.5-0 \mathrm{~g}$ ): this difference was significant $(P<0 \cdot 001)$. 
The peak scores of the birds in the two groups were not significantly different. However, if the next 4 highest values for each bird were expressed as a percentage of the peak score large differences between the groups were apparent. In Group 1 the median amounts placed on days ranked 2-5 were at least $50 \%$ of the highest score, while in Group 2 these scores were all less than $50 \%$. These differences were all significant (Table 2).

Table 2. Placing scores (ranked 2-5), shown as medians and interquartile ranges of the percentages of the peak placing score (rank 1), of nest material by oestrogen-treated ovariectomized canaries (7/group) for which the nest material was either removed daily (Group 1) or left undisturbed (Group 2)

\begin{tabular}{cllc}
\hline & Group 1 & Group 2 & Significance \\
\hline Peak placing score (g) Rank 1 & $21(30-6)$ & $12(21-9)$ & N.S. \\
Rank 2 $\%$ ) & $90(95-76)$ & $46(66-25)$ & $P<0.005$ \\
Rank 3 $\%)$ & $69(79-37)$ & $27(33-15)$ & $P=0.01$ \\
Rank 4 $(\%)$ & $62(79-29)$ & $17(28-6)$ & $P=0.01$ \\
Rank 5 $\%$ (\%) & $50(72-11)$ & $5(24-0.3)$ & $P<0.05$ \\
\hline
\end{tabular}

A bird was considered to be incubating if it was seen sitting low in the nest without making obvious nest-building movements; such birds are often very resistant to disturbance and will not move even when the experimenter approaches. When a bird was seen sitting for 2 or more consecutive days it was recorded as having reached the incubation criterion. Three birds reached this criterion; these were all in Group 2 and had already built nests. Between Days 15 and 33 they were seen sitting on 20,38 and $55 \%$ of 89 observations made during the first $8 \mathrm{~h}$ of the 14-h day. One other bird in this group and two in Group 1 were seen to sit but each did so on 1 day only. The decrease in building behaviour could not have been a direct consequence of incubation as it decreased in those birds that did not incubate as well as in those that did.

These results show that continuous high levels of circulating oestrogen are not incompatible with a decline in nest-building or with the appearance of incubation behaviour. It is therefore possible that, in intact birds, stimuli from the nest, or received in the course of building, exert an inhibitory effect on nest-building not (or not only) by reducing ovarian oestrogen output but by interfering with the effectiveness of the steroid in inducing the behaviour. If the nest material is removed from the nest pan this inhibitory effect is largely dissipated over $24 \mathrm{~h}$, but the continued presence of the nest and/or contact of the birds with it prolongs the inhibition of building behaviour.

\section{References}

HINDE, R.A. (1958) The nest-building behaviour of domesticated canaries. Proc. zool. Soc. Lond. 131, $1-48$.

Hinde, R.A. (1962) Temporal relations of brood patch development in domesticated canaries. Ibis 104, 90-97.

Hinde, R.A. (1965) Interaction of internal and external factors in integration of canary reproduction. In Sex and Behavior, pp. 381-415. Ed. F. Beach. Wiley and Sons, New York.

HiNde, R.A. \& STEEL, E. (1976) The effect of male song on an oestrogen-dependent behaviour pattern in the female canary (Serinus canarius). Horm. \& Behav. 7, (in press).

Hinde, R.A., Bell, R.Q. \& Steel, E.A. (1963) Changes in sensitivity of the canary brood patch during the natural breeding season. Anim. Behav. 11, 553-560.

Hinde, R.A., Steel, E. \& Follett, B.K. (1974) Effect of photoperiod on oestrogen-induced nest-building in ovariectomized or refractory female canaries (Serinus canarius). J. Reprod. Fert. 40, 383-399.

WARREN, R.P. \& Hinde, R.A. (1959) The effect of oestrogen and progesterone on the nest-building of domesticated canaries. Anim. Behav. 7, 209-213. 